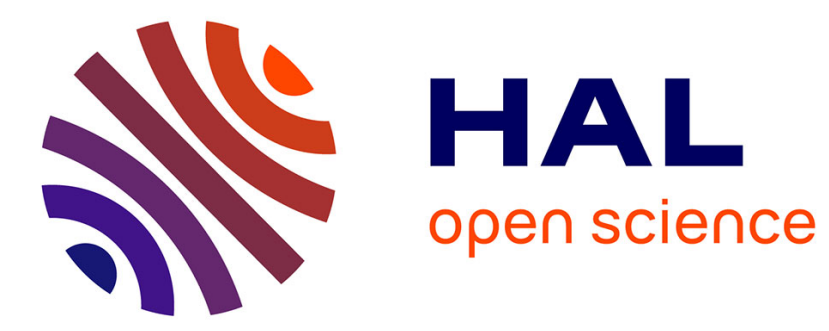

\title{
A Tetrabenzophenazine Low Voltage Single Molecule XOR Quantum Hamiltonian Logic Gate
}

\author{
We -Hyo Soe, Carlos Manzano, Christian Joachim
}

\section{To cite this version:}

We -Hyo Soe, Carlos Manzano, Christian Joachim. A Tetrabenzophenazine Low Voltage Single Molecule XOR Quantum Hamiltonian Logic Gate. Chemical Physics Letters, 2020. hal-03022376

\section{HAL Id: hal-03022376 https://hal.science/hal-03022376}

Submitted on 24 Nov 2020

HAL is a multi-disciplinary open access archive for the deposit and dissemination of scientific research documents, whether they are published or not. The documents may come from teaching and research institutions in France or abroad, or from public or private research centers.
L'archive ouverte pluridisciplinaire HAL, est destinée au dépôt et à la diffusion de documents scientifiques de niveau recherche, publiés ou non, émanant des établissements d'enseignement et de recherche français ou étrangers, des laboratoires publics ou privés. 


\section{A Tetrabenzophenazine Low Voltage Single Molecule XOR Quantum Hamiltonian Logic Gate}

We-Hyo Soe, ${ }^{a, c, *}$ Carlos Manzano ${ }^{b}$ and Christian Joachim ${ }^{a, c}$

${ }^{a}$ CEMES, Université de Toulouse, CNRS, 29 Rue J. Marvig, BP 94347, 31055 Toulouse Cedex, France

${ }^{\mathrm{b}}$ Institute of Material Research and Engineering (IMRE), 3 Research Link, Singapore, 117602, Singapore

c International Center for Materials Nanoarchitectonics (WPI-MANA), National Institute for Material Sciences (NIMS), 1-1 Namiki, Tsukuba, Ibaraki 305-0044, Japan

\section{abstract}

Herein it is demonstrated that a tetrabenzo[a,c,j.h]phenazine molecule is able to function like a " 2 input - 1 output" XOR Boolean logic gate due to the quantum level repulsion effect which results from the logic gate's Quantum Hamiltonian Computing design. The logical inputs are performed using one aluminium atom per input. The logical output is measured by recording the $\mathrm{dl} / \mathrm{dV}$ tunneling electronic spectrum at a low $+1.1 \mathrm{~V}$ bias voltage. On an $\mathrm{Au}(111)$ surface, each $\mathrm{Al}$ atom is manipulated in and out of the molecule by scanning tunneling microscope single molecule manipulations. This single molecule XOR gate functions without the need of cascading OR, AND and NAND logic gates inside its molecular structure. 


\section{Introduction}

A single molecule logic gate is a conjugated molecule able alone to compute or process a logical decision for example to perform a Boolean logical operation. Its chemical design can be classical or quantum [1-3]. In a classical design, its molecular structure mimics the electrical network of an electronic circuit by cascading molecular wires, rectifiers and switches, all bonded together to form a single molecule: the molecular circuit [4]. However this architecture has its drawbacks due the exponential decay of the current intensity along the molecular circuit, such decay increases with the length of the molecular components hence constraining the implementation of a molecular network circuit [5]. A quantum molecule logic gate can be designed with or (more recently) without qubits $[6,7]$. Nevertheless using qubits in a molecule [3] also limits the complexity of the Boolean functions to be performed since the intramolecular qubits must be located far away from each other along the molecular structure to limit inter qubits through bond electron transfer processes. The quantum Hamiltonian computing (QHC) approach was proposed as an alternative to surmount this problem [6,8]. QHC is a qubit free quantum computing approach where the Boolean logic functioning is ensured by the quantum level repulsion effects [7]. Classical logical inputs are here performed by directly perturbing the molecular electronic structure for example by bringing single metallic atoms in interaction with the calculating molecular board $[9,10]$ or by switching chemical groups directly bonded to this board [11]. The logical outputs are measured locally on the molecular board without the need to pass a current through the complete molecular structure $[9,10]$.

The universal quantum graph of the basic but fundamental "2 inputs -1 output" QHC logic gate is now known together with more complex QHC graphs like the one for the 2 digits Boolean $1 / 2$ adder $[7,11,12]$. A molecule from the starphene series has been shown to be able to perform a QHC NOR logical operation [9]. More recently, a QHC NAND was obtained with another molecule of the same series [10]. A QHC NOR atomic scale circuit was also recently constructed atom by atom on the $\mathrm{Si}(100) \mathrm{H}$ surface [13], an encouraging step towards the development and implementation of QHC logic gates using well stablished surface semiconductor technology. In QHC, there is also no direct relation between a given level of quantum graph complexity and the next level of complexity for Boolean logic. For example, the QHC XOR quantum graph has the same number of quantum states than a NOR [12] while in classical electronic circuits, a XOR gate 
requires an OR gate cascaded with AND and NAND gates. Such cascading is not required in the QHC approach.

In this letter it is demonstrated that a molecule XOR gate can function without cascading elementary OR, AND and NAND logic gates inside the same molecular structure, we report the functioning of a QHC XOR single molecule. Using a low temperature scanning tunnelling microscope (LT-UHV-STM), this gate is operated by manipulating single Al atoms for inputting the logical data and by measuring its local $\mathrm{dl} / \mathrm{dV}$ electronic spectra as a function of the logical input configurations. In section 2 , the design strategy leading to a tetrabenzo[a,c,j.h]phenazine is shortly described. In section 3, the sublimation of the molecules on the Au(111) surface and the deposition of single Al atoms is described and LT-UHV-STM images presented. In section 4, LTUHV-STM dl/dV spectra of a bare tetrabenzo[a,c,j.h]phenazine and the metal organic complexes formed upon the coordination of $\mathrm{Al}$ atoms are presented and discussed. The tetrabenzo[a,c,j.h]phenazine XOR Boolean logical functioning is discussed in section 5 before the conclusion.

\section{The choice of the tetrabenzo[a,c,j.h]phenazine molecule}

Starting with an anthracene molecule physisorbed on an Au(111) surface, single metal atoms can certainly be STM manipulated towards and brought in contact in a symmetric way with the anthracene's central phenyl. Such interaction will result in an energy shift of the molecule's electronic states which can be tracked using dl/dV LT-UHV-STM spectroscopy and mapping $[9,10]$. The voltage range accessible experimentally for this tracking without damaging the molecule is limited to a window of $\pm 3 \mathrm{~V}$. However anthracene is too small to accommodate at the same time 2 metal atoms in a way to set up independently a logical $(1,1)$ input as performed for the QHC NOR starphene input operations [9]. Adding 4 phenyls in a symmetric way around the anthracene will help to stabilize the $(1,1)$ input configuration leading to the tribenzotetraphene molecule whose structure is shown in the Figure 1a. Similar to what was proposed in [14] when the two $\mathrm{CH}$ of the central phenyl are substituted by two methylene groups (Figure 1b), this very local chemical transformation from a sp2 to a sp3 configuration gives rise to a change in the tribenzo-tetraphene electronic structure. Its lowest unoccupied molecular orbital 
(LUMO) and its LUMO+1 are downshifted becoming now respectively the highest occupied molecular orbital (HOMO) and LUMO of this new dihydroanthracene-tetraphene molecule. Some other tribenzo-tetraphene MOs like HOMO-1 do not shift in energy after this chemical transformation. Since the tribenzo-tetraphene LUMO+1 has a central nodal plane and changing from tribenzo-tetraphene to dihydroanthracene-tetraphene, this nodal plane is now accessible but down in energy. In terms of a Boolean logical output, this means that by positioning the STM tip apex on this nodal plan, no tunnelling current will flow through the dihydroanthracenetetraphene molecule while at the same location, a current will flow through the tribenzotetraphene. This is exactly how a QHC logic gate is supposed to operate when measured by STM $[9,10]$.

To complete our design and since the sp2 to sp3 local chemical transformation described above from tribenzo-tetraphene to dihydroanthracene-tetraphene would not be reversible through the STM manipulation of atom inputs, the two involved central carbons on the tribenzo-tetraphene central phenyl have been substituted by nitrogen atoms leading tetrabenzo[a,c,j.h]phenazine molecule, see Figure 1c.

Aluminum atoms were selected to be used as the logic gate inputs because an Al ad-atom chemisorbed on $\mathrm{Au}(111)$ is generally found in an intermediate oxidation state between $\mathrm{Al}(\mathrm{I})$ and Al(II) [15]. To perform a logical input, this enables a directional coordination of a single Al adatom with one of the two nitrogen lone pairs of this molecule on the Au(111) surface. Figure 1c and $1 \mathrm{~d}$ are presenting the calculated MOs of respectively the tetrabenzo-[a,c,j.h]phenazine and its $\mathrm{Al}_{2}$-molecule complex [16]. Similarly to the discussion above regarding the electronic state shifts arising from redesigning tribenzo-tetraphene molecule with methylene, the $\mathrm{Al}_{2}$-complex MOs are also shifted down in energy as compared with the tetrabenzo[a,c,j.h]phenazine alone. The tetrabenzo[a,c,j.h]phenazine MO nodal structure can be easily recognized at lower energies in the $\mathrm{Al}_{2}$-complex $\mathrm{MOs}$ as presented Figure $1 \mathrm{~d}$. For instance as a result of this energy shifts, tetrabenzo[a,c,j.h]phenazine's LUMO+1 is now the $\mathrm{Al}_{2}$-molecule complex's LUMO and the tetrabenzo[a,c,j.h]phenazine LUMO becomes the $\mathrm{Al}_{2}$-complex HOMO. This $\mathrm{MO}$ reorganization and energy shift are accompanied by a preservation of their central nodal plane required for the QHC logical operation. 


\section{Experimental implementation on the Au(111) surface}

Ultrapure powder of tetrabenzo[a,c,j.h]phenazine molecules was synthesized [17] and then sublimated using free evaporation by heating a quartz crucible at $240{ }^{\circ} \mathrm{C}$. The evaporation parameters were selected to deposit a minute amount of molecules. Such deposition conditions lead to a sub-monolayer coverage of the $A u(111)$ surface which is important to avoid molecular clustering as well as to provide enough space in between the deposited Al atoms and molecules to perform the STM molecule manipulations. The molecules were deposited on an $\mathrm{Au}(111)$ single-crystal which was cleaned using standard methods consisting of several cycles of Ar+ ion sputtering and subsequent annealing. During the molecule deposition, the gold substrate temperature was kept below $40{ }^{\circ} \mathrm{C}$. The sample was cooled down to liquid helium temperature before its transfer to the STM chamber. The aluminium atom source was mounted on the STM chamber to deposit Al single atoms on the Au(111) surface. During this deposition, the substrate was kept at cryogenic temperatures. All STM imaging, tunnelling spectroscopy measurements and molecule manipulations were done at $\sim \mathrm{K}$ using a Createc LT-UHV-STM.

Using the STM molecule manipulation technique, the step by step process of a single onsurface $\mathrm{Al}_{2}$-complex construction is presented in Reference 15 . Experimental work done to develop metal-molecule complex revealed that a single Al atom is invariably going to bind to one of the central pyrazine's nitrogen atoms as anticipated by design. Once an Al atom is coordinated with the molecule having the shape of a letter ' $\mathrm{H}$ ', the centre of this on-surface complex is imaged with a bright contrast as compared with centre of a bare molecule (see topographic images in Figures $2 \mathrm{a}$ and $2 \mathrm{~b}$ ). When a second $\mathrm{Al}$ atom is complexed with the other nitrogen, the central part of this new complex appears even brighter than $\mathrm{Al}_{1}$-complex (topographic image in Figure 2c).

\section{LT-UHV-STM dI/dV Spectroscopy}

The molecular electronic structures of $\mathrm{Al}_{0^{-}}, \mathrm{Al}_{1^{-}}$and $\mathrm{Al}_{2}$-molecule complexes were assessed using scanning tunnelling spectroscopy (STS). The STS spectra were first recorded at 3 different locations along the $\mathrm{H}$-molecule, the spectra capturing the most significant signatures are presented in Figure 2a. They reveal 4 electronic tunnelling resonances, 2 at negative and 2 at 
positive bias voltages which have been identified respectively as the 1 st and 2 nd oxidized $\& 1$ st and 2 nd reduced states tunnelling resonances. The differential conductance (dl/dV) maps recorded at these 4 resonances bias voltages are presented Figures $2 a(i-i v)$. The dl/dV map corresponding to the 1st negative resonance (Figure 2a(ii)), looks like made of two horseshoelike lobes facing one above the other. No internal structure is imaged. Likewise, the 2nd oxidized state map does not show any internal features. Only peanut like lobes appear at the right and left sides of the molecule, see Figure $2 \mathrm{a}(\mathrm{i})$. The most significant features imaged at the $1 \mathrm{st} \mathrm{dl} / \mathrm{dV}$ positive resonance map are the two bright lobes prominently arising at each side of the central pyrazine as can be seen in Figure $2 \mathrm{a}(\mathrm{iii})$. The 2 nd reduced state $\mathrm{dl} / \mathrm{dV}$ map is like the negative contrast image of the 1st reduced state map (see Figure 2a(iv)), where the previously imaged bright lobes at the sides of the molecule centre show up as low differential conductance lobes. The corresponding images calculated using mono-electronic elastic scattering quantum chemistry (ESQC) [18] are presented for comparison Figures 2a(v-viii). Aside from the LUMO, they reproduce quite well the main experimental features. The HOMO and HOMO-1 contributions to the 1 st and 2 nd resonances at negative voltage are quite clear together with the LUMO+1 contribution to the 2 nd resonance at positive bias voltage. The map of the resonance recorded around $2 \mathrm{~V}$ is not well reproduced by the mono-electronic approximation used in ESQC indicating that not only the LUMO is contributing to the through molecule electron transfer process building up this resonance.

The spectra recorded on the $\mathrm{Al}_{1}$-molecule complex formed after the STM manipulation of one Al towards the central pyrazine (see Figure $2 b$ ) demonstrate that the tunnelling resonances identified as the 1 st and 2 nd reduced states of $\mathrm{H}$-molecule are now downshifted towards the substrate's Fermi level by about $800 \mathrm{mV}$ and $400 \mathrm{mV}$ respectively. Moreover, the intensity of the oxidized state tunnelling resonances has decreased as compared to the original bare molecule's ones and the 1st resonance seems to be totally screened by the metal surface.

Although the energy shifts for both reduced state resonances are large, the effects of the Al coordination on $\mathrm{Al}_{1}$-complex $\mathrm{dl} / \mathrm{dV}$ maps is quite small indicating that the LUMO and LUMO+1 quantum weight contributions to those resonances is quite stable. Here, the single Al was complexed on the right side as indicated by the green dot on the STM topographic image. The central right lobes at the nitrogen vicinity are still imaged with a high differential conductance and show a higher contrast than on rest of the molecule. The consequences of the Al 
coordination are more evident on the 2 nd reduced state $\mathrm{dl} / \mathrm{dV}$ map. Before the complexation, two dark contrast lobes are observed at both sides of the molecule's centre. After one Al contact, the lobe on the Al-N coordination bond is slightly changed from a dark to a bright contrast while on the opposite un-coordinated side, its dark contrast lobe is kept similar to the one on a free H-molecule's STM image (see Figure $2 \mathrm{~b}(\mathrm{ii})$ ).

When a second $\mathrm{Al}$ is manipulated to form the $\mathrm{Al}_{2}$-molecule complex, as presented Figure $2 \mathrm{c}$, its spectrum recorded at the Al-N site (green line) presents no resonance at positive bias voltage in contrast to the peak appearing within the same voltage range in spectra from $\mathrm{Al}_{0^{-}}$and $\mathrm{Al}_{1^{-}}$ complexes. The $\mathrm{Al}_{2}$-molecule complex reduced states are now only captured at the corner of molecule. Moreover, the down oxidized state resonances are screened under a broad band tunnelling resonance tail.

The 1 st reduced state $\mathrm{dl} / \mathrm{dV}$ map of this $\mathrm{Al}_{2}$-complex is presented in Figure $2 \mathrm{c}(\mathrm{i})$. This map allows to identify the resonance as corresponding to the 2nd reduced states of both the bare $\mathrm{Al}_{0^{-}}$and the $\mathrm{Al}_{1}$-complexes. This $\mathrm{dl} / \mathrm{dV}$ image also shows the same characteristic ' $\mathrm{H}^{\prime}$ shape observed before for 2 nd reduced state maps. However, two additional lobes appear at the centre of the $\mathrm{Al}_{2}-$ molecule in the vicinity of the two Al-N bonds. The identification of this resonance makes clear that by contacting a second $\mathrm{Al}$ to $\mathrm{Al}_{1}$-molecule complex, its reduced states shift again in energy by more than $600 \mathrm{mV}$ towards Au(111) Fermi Level. Intuitively the resonance inherited from the $\mathrm{Al}_{1}$-complex 1 st reduced state should have also been shifted too. None of the $\mathrm{Al}_{2}$-molecule complex spectra present a trace of such a resonance.

The absence of this tunnelling resonance can be explained by considering again the calculated $\mathrm{MO}$ of the bare- and the $\mathrm{Al}_{2}$-molecule presented in Figure 1. When the $\mathrm{Al}$ are coordinated to the two-central pyrazine nitrogen's, the MO lobes originally located on the nitrogen change their spatial hybridization from sp2 to sp3. The $\mathrm{Al}_{2}$-complex $\mathrm{MO}$ are shifted to lower energy with respect to the bare $\mathrm{H}$-molecule MO. More importantly, the H-molecule's LUMO becomes the $\mathrm{Al}_{2}$-molecule complex $\mathrm{HOMO}$. The missing resonance of $\mathrm{Al}_{2}$-complex was certainly shifted down to the $A u(111)$ Fermi level and becomes very sharp [15].

\section{The QHC XOR Logic gate}


The Al atoms manipulation towards the tetrabenzo[a,c,j.h]phenazine are the classical logical inputs of our QHC XOR logic gate. One coordinated Al is encoding a logical input " 1 ". With its 2 nitrogen central atoms and its structural symmetry, the H-molecule can accommodate the $(0,0)$, $(0,1),(1,0)$ and $(1,1)$ logical inputs required for a two logical inputs XOR Boolean logic gate.

The QHC logical operation occurs when one Al is STM manipulated an brought into electronic interactions with the central pyrazine ring of the molecule. This input operation is a nice example of a classical to quantum information conversion. The classical action of laterally manipulating one $\mathrm{Al}$ with the STM tip on the $\mathrm{Au}(111)$ surface ends when the $\mathrm{Al}_{\mathrm{X}}$-molecule complex $(x=0,1,2)$ is stabilized. Here, the classical information: "an Al atom is in or out of the molecule" is converted in a change of the molecule MO weights decomposed for example on a Slater atomic orbital basis set. It carries the quantum information on how many Al are complexed. After one Al atom contact, the down shift of the first positive resonance of the molecule from $+1.9 \mathrm{~V}$ to $1.1 \mathrm{~V}$ and the corresponding reorganization of the $\mathrm{H}$-molecule electronic cloud as mapped using the $d l / d V$ technique is the measurement of this weight redistribution. It indicates how the molecule is taking into-account one or two Al atoms. It answers by a specific intrinsic electronic reorganization leading to the QHC logic gate functioning.

The reading of the logical output status of our gate is obtained by positioning the STM tip apex exactly at one nitrogen location on the $\mathrm{H}$-molecule and by selecting the measuring bias voltage. In QHC, different Boolean truth table can be found for different output reading energies [7]. As presented in Figure $3 c$, the $+1.1 \mathrm{~V}$ resonance is existing when only one $\mathrm{Al}$ is interacting with the central pyrazine corresponding to the $(0,1)$ and $(1,0)$ logical inputs. For two Al atoms in contact (the $(1,1)$ logical input), there is no resonance at $+1.1 \mathrm{~V}$. For the $(0,0)$ logical input (the $\mathrm{H}$ molecule with no complexed $\mathrm{Al}$ ), there is also no resonance at $+1.1 \mathrm{~V}$ since its first positive resonance is located at $+1.9 \mathrm{~V}$. As presented in the table Figure $3 \mathrm{~b}$ and for this $+1.1 \mathrm{~V}$ output reference voltage, this is the functioning of a Boolean XOR gate. In this STS spectroscopy, the ON/OFF ratio between a logical output " 0 " and " 1 " is reasonable. With the tip apex exactly located on one central nitrogen, as presented in Figure 3d, the STM junction I-V curves present also a large margin between the ON (configurations $(0,1)$ or $(1,0)$ ) and OFF (configurations $(0,0)$ or $(1,1))$ current intensities, $0.2 \mathrm{nA}$ and $0.09 \mathrm{nA}$ respectively for a $1.55 \mathrm{~V}$ bias voltage. Notice also that $+1.9 \mathrm{~V}$ can be selected as a reference output voltage for the gate. According to the above 
discussion, this will lead to a QHC NOR gate but with an operation voltage close to the stability limit of the $A l_{x}$-molecule complex in the STM junction. Hence showing that the tetrabenzo[a,c,j.h]phenazine molecule functions in parallel as an XOR and a NOR logic gate.

\section{Conclusion}

We have demonstrated that a small tetrabenzo[a,c,j.h]phenazine molecule can perform an XOR Boolean logic function at a low $+1.1 \mathrm{~V}$ measuring voltage without the need to cascade OR, AND and NAND logic gates inside the molecule as usually performed in electronic circuits. The molecular structure has the same size than the starphene which is only performing a NOR function. With quantum computing circuits using a set of qubits [3], the quantum trajectory of the logic gate during its time dependent operation is also well controlled but at the expense of a large increase of the number of qubits to physically implement more and more complex logic gates [19]. We are confident that a QHC molecule can also operate complex Boolean logical operations with only a modest lateral increase of its physical dimensions.

\section{Acknowledgements}

We thank Prof. Antonio M. Echavarren (Institute of Chemical Research of Catalonia, Spain) and Prof. Claudia de León (Universidad Mariano Gálvez, Guatemala) for having kindly provided the tetrabenzo[a,c,j,h]phenazine molecule, and acknowledge the Agency of Science, Technology and Research (A*STAR), Singapore - the Visiting Investigatorship Program: Atom Tech project (2005-2014) and the European Commission integrated project AtMol for financial support.

\section{References}

[1] F. L. Carter, Physica D 10 (1984) 175.

[2] A. Aviram, J. Am. Chem. Soc. 110 (1988) 5687-5692. 
[3] M. A. Nielsen, I. L. Chuang, Quantum Computation and Quantum Information (Cambridge University Press, Cambridge, 2000)

[4] J. C. Ellenbogen, J. C. Love, Proc. IEEE 88 (2000) 386.

[5] C. Nacci, F. Ample, D. Bleger, S. Hecht, C. Joachim, L. Grill, Nat. Comm. 6 (2015) 7397.

[6] C. Joachim, N. Renaud, M. Hliwa, Adv. Mater. 24 (2012) 312.

[7] O. Faizy Namarvar, O. Giraud, B. Georgeot, C. Joachim, Quantum Sci. Technol. 4 (2019) 035009.

[8] N. Renaud, C. Joachim, Phys. Rev. A 78 (2008) 062316.

[9] W.-H. Soe, C. Manzano, N. Renaud, P. De Mendoza, A. De Sarkar, F. Ample, M. M.Hliwa, A. M. Echavarren, N. Chandrasekhar, C. Joachim, ACS Nano 5 (2011) 1436.

[10] D. Skidin, O. Faizy, J. Krüger, F. Eisenhut, A. Jancarik, K. H. Nguyen, G. Cuniberti, A. Gourdon , F. Moresco, C. Joachim, ACS Nano 12 (2018) 1139.

[11] S. Srivastava, H. Kino, C. Joachim, Chem. Phys. Lett. 667 (2017) 301.

[12] G. Dridi, R. Julien, M. Hliwa, C. Joachim, Nanotechnology, 26 (2015) 344003.

[13] M. Kolmer, R. Zuzak, S. Godlewski, M. Szymonski, G. Dridi, C. Joachim, Nanoscale, 7 (2015) 12325.

[14] P. W. K. Jensen, C. Jin, P. L. Dellaire-Demers, A. Aspuru-Guzik, G. C. Solomon, Quantum Sci. Technol. 4 (2019) 015013.

[15] W.-H. Soe, C. Manzano, R. Robles, N. Lorente, C. Joachim, Nano lett. 20 (2020) 384.

[16] M. W. Schmidt, K. K. Baldridge, J. A. Boatz, S. T. Elbert, M. S. Gordon, J. H. Jensen, S. Koseki, N. Matsunaga, K. A. Nguyen, S. J. Su, T. L. Windus, M. Dupuis, J. A. Montgomery, J. Comput. Chem. 14 (1993) 1347.

[17] B. Schidt, Prakt. Chem., 1941, 157, 203; J. Bolger, A. Gourdon, E. Ishow, J. -P. Launay, J. Inorg. Chem. 35 (1996) 2937.

[18] P. Sautet, C. Joachim, Chem. Phys. Lett. 185 (1991) 23.

[19] V. Vedral, A. Barenco, A. Ekert, Phys. Rev. A, 54 (1996) 147. 
(a)

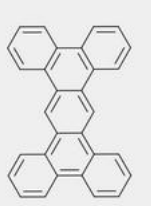

(b)
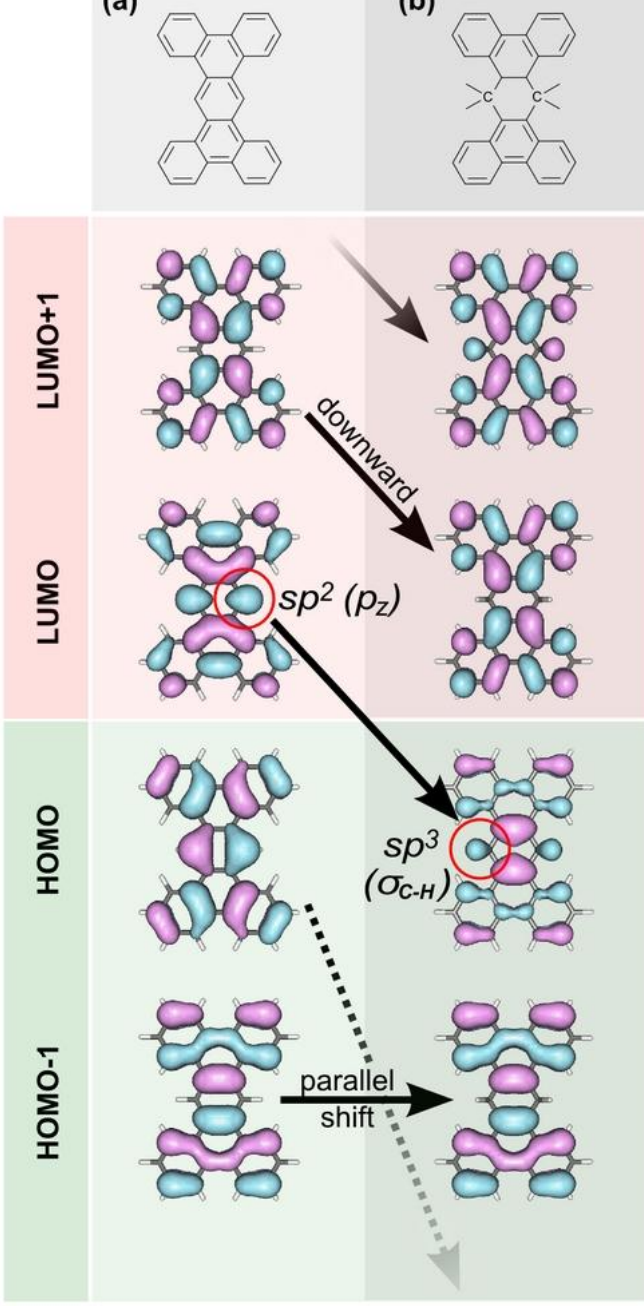

(c)

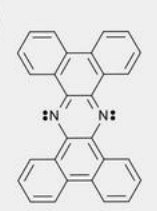

(d)
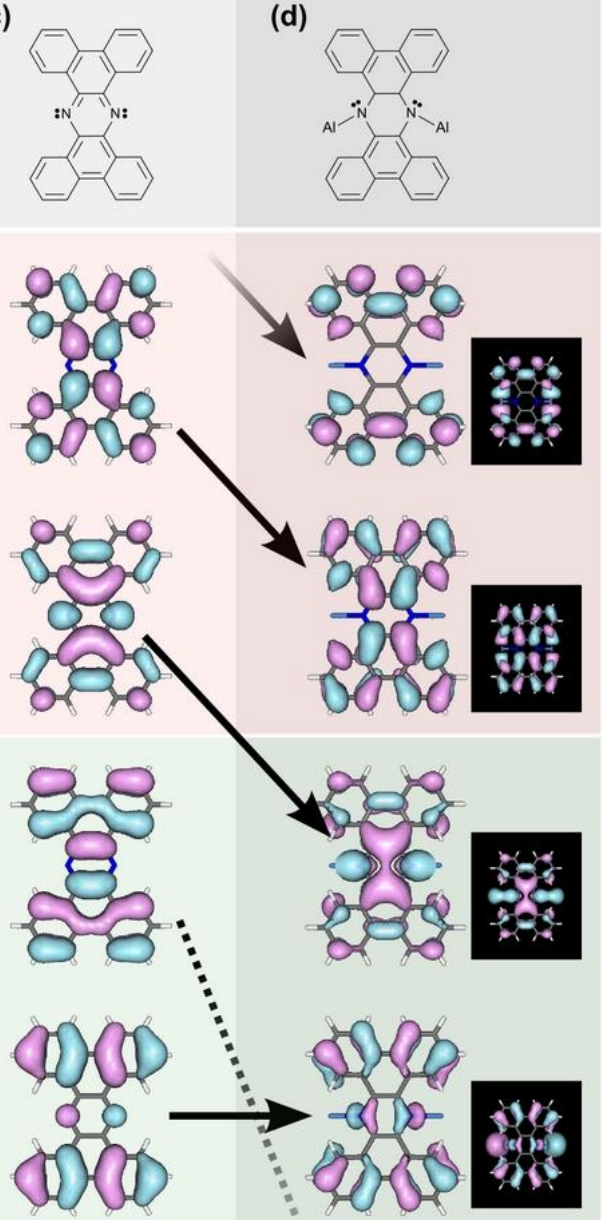

Figure 1. Computed images of the frontier molecular orbitals (MOs) of the four molecules used to illustrate the QHC level repulsion effect and their chemical structure (first row). (a) The starting tribenzo-tetraphene molecule where HOMO-1 and LUMO+1 have no contribution on the central $\mathrm{CH}$. (b) The dihydroanthracene-tetraphene molecule where those two $\mathrm{CH}$ have been substituted by methylene groups. Here, HOMO and LUMO shift up in between the tribenzo-tetraphene LUMO and LUMO+1 because of the change from sp2 to sp3 orbitals. This level repulsion effect is indicated by the arrows from (a) and (b). (c) The tetrabenzo[a,c,j.h]phenazine molecule has two central nitrogen atoms. Its frontier MO are almost the same as those from the original tribenzo-tetraphene. But its HOMO and HOMO-1 appear instead in an inverted order with respect to the original tribenzo-tetraphene because of the nitrogen lone-pair. (d) With its two coordinated $\mathrm{Al}$, the $\mathrm{Al}_{2}$-molecule complex brings the same repulsion level effect taking place when modifying a tribenzo-tetraphene to dihydroanthracene-tetraphene. This shift is indicated by the arrows from (c) and (d). Column $d$ insert: The MO spatial extension arising from the complexed Al significantly overshadows the contributions of neighboring nodal lobes of each MO making difficult to correlate the bare $\mathrm{H}$-molecule to $\mathrm{Al}_{2}$-molecule complex $\mathrm{MO}$ shifts in energy. To ease this identification, the column d MOs have been adjusted in electron density in the inserts to diminish the screening introduced by the Al contributions. Calculations were performed using GAMESS an ab initio quantum chemistry program [16]. 


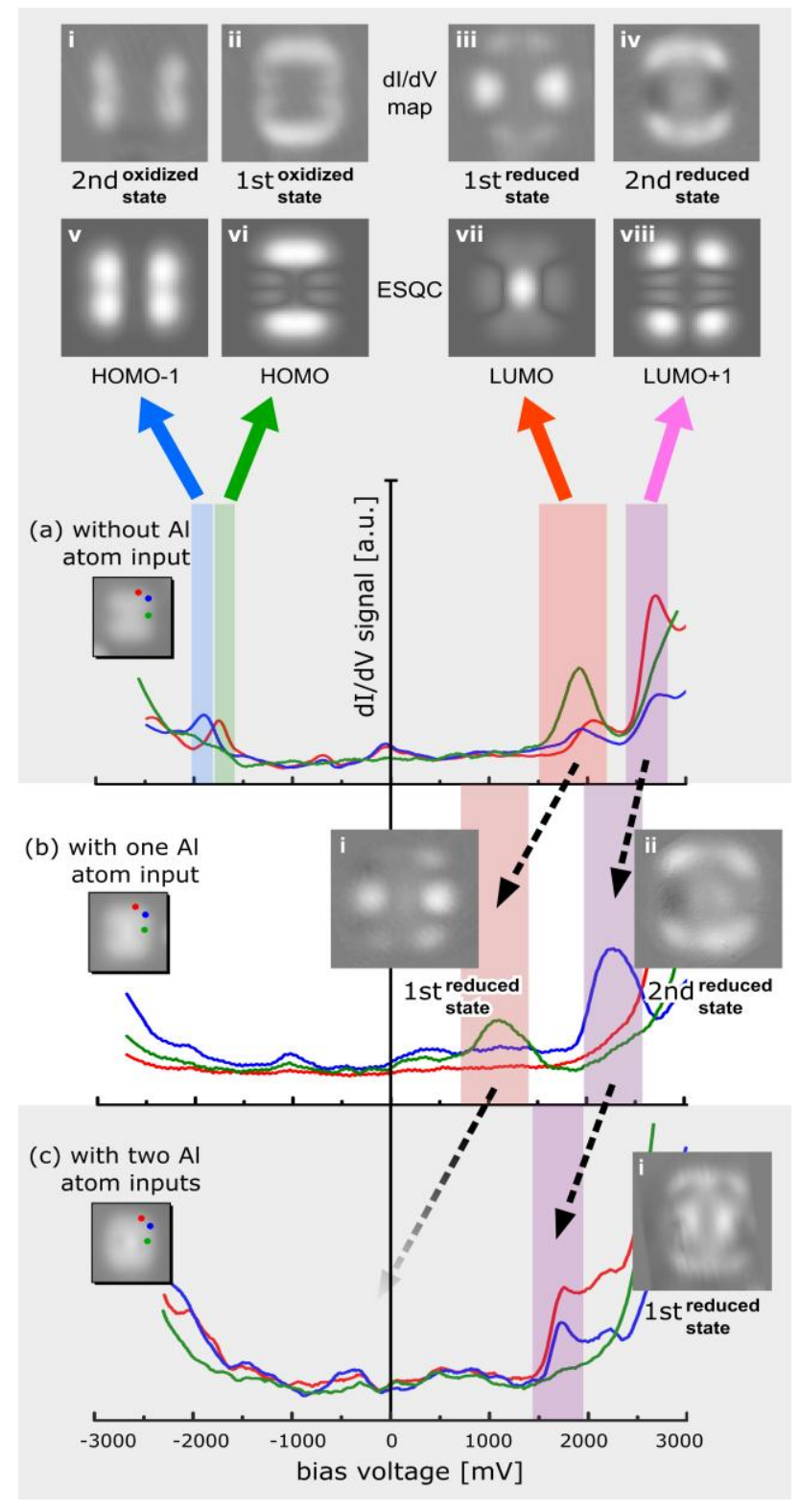

Figure 2. Scanning tunnelling spectroscopic measurements of the QHC XOR logic gate molecule. (a) dl/dV spectra of a free $\mathrm{H}$-molecule recorded at three different tip locations (The spectrum line colours are corresponding to the tip's position indicated by coloured dots in the topographic image on the left). Images a(i-vi) are the experimental $\mathrm{dl} / \mathrm{dV}$ differential conductance maps recorded at tunnelling resonant for both bias voltage polarities. Images a(vviii) are the corresponding calculated ESQC maps. In the dl/dV spectra of the bare molecule four resonances are captured within the bias voltage range measured. (b) $\mathrm{dl} / \mathrm{dV}$ spectra of the constructed $\mathrm{Al}_{1}$-molecule complex taken at the same STM tip positions. Two tunnel resonances remain at positive bias voltage while at the negative side, the resonances amplitude decrease drastically. Images $\mathrm{b}(\mathrm{i}-\mathrm{ii})$ are the experimental $\mathrm{dl} / \mathrm{dV}$ maps recorded at the two positive resonances. Each map is very similar to the corresponding one of the free molecules in a(iii-iV). (c) $\mathrm{dl} / \mathrm{dV}$ spectra of the $\mathrm{Al}_{2}$-molecule complex. No resonance is captured at bias voltages below $+1.50 \mathrm{~V}$. The first resonance is showing up around $+1.70 \mathrm{~V}$. The differential conductance map of this resonance inherits its 
characteristics from the second resonance (positive) of the free $\mathrm{H}$-molecule, shown in a(iv). All image sizes are 2.5 $\mathrm{nm} \times 2.5 \mathrm{~nm}$.

(a)

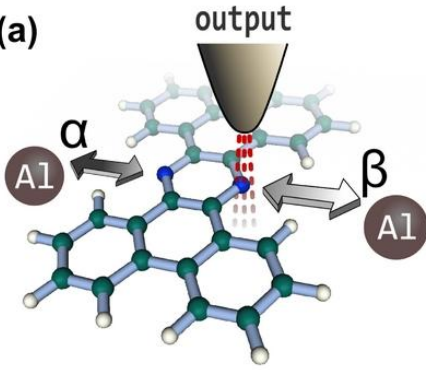

(b)

\begin{tabular}{|c|c|c|c|c|}
\hline $\begin{array}{r}\text { input } \\
\text { output }(\alpha, \beta)\end{array}$ & $(0,0)$ & $(0,1)$ & $(1,0)$ & $(1,1)$ \\
\hline$\alpha \oplus \beta$ & 0 & 1 & 1 & 0 \\
\hline $\mathrm{dl} / \mathrm{dV} V_{V=1.10 V}$ & 0.40 & 1.0 & 1.0 & 0.08 \\
\hline $\mathrm{I}_{V=1.55 V}$ & 0.55 & 1.0 & 1.0 & 0.48 \\
\hline
\end{tabular}
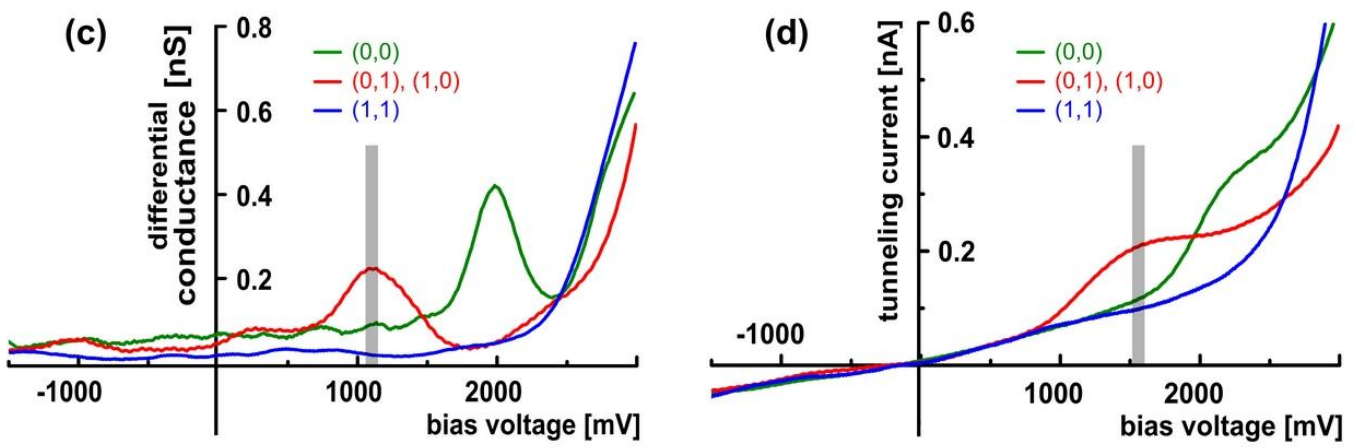

Figure 3. (a) Schematics of the QHC XOR logic gate where the "tip-nitrogen atom-gold surface" tunnel junction is used to measure the logic gate output status via the $\mathrm{dl} / \mathrm{dV}$ resonance shift or directly using the tunnelling current intensity. (b) The XOR truth table with the corresponding $\mathrm{dl} / \mathrm{dV}$ (3rd row) and current intensity values (4th row). Differential conductance intensity re-normalized using the conductance at $\mathrm{V}=1.10 \mathrm{~V}$ and current intensity using the intensity at $V=1.55 \mathrm{~V}$ for the $(0,1)$ input configuration in both cases. (c) For each input configuration in Figure $2 a, 2 b$ and $2 c$, zoom-in $d l / d V$ spectra picking-up only the spectrum recorded with the tip apex above the nitrogen (green-dotted position in Figure 2 topographic image), (d) The corresponding I-V characteristics. 